\title{
L'importance de préserver la diversité des accents pour contrer l'insécurité linguistique en Ontario français
}

\author{
Christian Bergeron \\ Université d'Ottawa
}

\section{Introduction}

Le Canada est un pays enrichi par le multiculturalisme et l'immigration, mais la protection et la valorisation de la langue française demeurent un enjeu majeur pour les communautés francophones hors Québec. Certains francophones luttent pour leur survie afin d'éviter l'assimilation au groupe dominant anglophone (Labrie), alors que d'autres s'accommodent de la situation linguistique majoritaire, tout en conservant une identité francophone jumelée à une identité anglophone (Vaillancourt). Or, même si les rapports sociaux sont généralement harmonieux entre les communautés francophones et anglophones, il existe des rapports de force entre elles. Les rapports de force sont ici compris dans le sens de Bourdieu, c'est-à-dire des «luttes pour le monopole du pouvoir de faire voir et de faire croire, de faire connaittre et de faire reconnaittre, d'imposer la définition légitime des divisions du monde social » (Ce que parler 137).

Le « choc des langues » au Canada ou ailleurs dans le monde témoigne d'un même phénomène social : " on ne peut couper le rapport de domination entre langues différentes de celui qui existe entre variétés d'une même langue» (Klinkenberg 37). D'une part, il y a l'hégémonie linguistique de la langue anglaise, incluant une propension chez les communautés francophones à utiliser l'anglais dans plusieurs contextes où le français devrait être plus présent (anglais parlé majoritairement dans les cours d'école de langue française, s'exprimer en anglais, alors que le service francophone existe, etc.). Cette forme de rapport entre les langues anglaise et française peut instaurer une forme d'insécurité statutaire (Calvet), c'est-à-dire une absence de reconnaissance publique de la langue française. D'autre part, un rapport de force existe à l'intérieur des communautés francophones au sujet de l'hégémonie idéologique d'un français normé qui amènerait les francophones à hiérarchiser les variétés de la langue parlée entre ceux qui maîtrisent (ou non) la langue française selon des normes subjectives établies (bon français, bon accent, absence d'anglicismes, etc.). En Ontario français, ces normes subjectives viennent très souvent du Québec (entre autres avec l'Office québécois de la langue française), mais aussi de la France pour les immigrants francophones (la norme française est fortement présente 
dans d'autres pays francophones). Cela dit, en général, la norme de la langue française en Ontario s'arrime majoritairement à celle du Québec, malgré les différences d'accents, de la langue parlée ainsi que des anglicismes. Ces deux formes de rapports de force, extrinsèques et intrinsèques aux communautés francophones, peuvent mener à des discriminations portant sur la langue française (Blanchet, Clerc et Rispail). Cette forme de discrimination linguistique, pour être plus précis la glottophobie (Blanchet), peut engendrer chez ceux qui la subissent une insécurité linguistique. En d'autres mots, il existe une forme de discrimination linguistique entre francophones au sujet du rejet d'une variation linguistique du français ancrée dans l'idéologie d'une norme linguistique unique (Dalley). Même si officiellement il n'y a pas une « norme reconnue » de la langue française en Ontario, à l'exception des emprunts à la norme québécoise, il existe tout de même dans les communautés francophones l'idéologie qu'il n'y aurait qu'un seul bon parler français, «un» et «indivisible» (Francard), mais qui amènerait certains locuteurs à ressentir, entre autres, de l'insécurité linguistique (Ce que parler, Brétégnier et Ledegen; LeBlanc). Selon le président de la Fédération de la jeunesse canadienne-française, Justin Johnson, « on vit de l'insécurité linguistique partout au pays, mais on n'a, surtout, pas les moyens de bien expliquer ce problème. On doit trouver des solutions et développer une stratégie nationale sur la sécurité linguistique » (cité dans Radio-Canada 2017, n. p.).

Dans le cadre de cet article, je traiterai de l'insécurité linguistique chez les communautés francophones ontariennes. Il est important de faire a priori un portrait général de l'Ontario francophone ainsi que du contexte de l'immigration canadienne afin de favoriser une compréhension partagée du contexte sociodémographique de la problématique abordée. Des données du recensement populationnel de Statistique Canada, effectué en 2016, seront utilisées pour documenter la province étudiée (Statistique Canada 2017a à 2017f; Statistique Canada 2016). Ensuite, à l'aide de la typologie de Calvet, à savoir l'insécurisation statutaire, formelle et identitaire, certains comportements épilinguistiques chez des francophones seront présentés et analysés, dont une capsule vidéo humoristique du groupe franco-ontarien Improtéine (intégrée à l'article : L'accent franco-ontarien standardisé). Précisons que

[...] les comportements dits épilinguistiques sont des manifestations implicites de l'idéologie linguistique : reprendre un enfant, réagir à une forme linguistique par le rire ou une mimique admirative, faire semblant de ne pas comprendre ou faire l'effort de comprendre une autre langue, choisir telle forme linguistique ou privilégier l'usage d'une langue, etc. (Blanchet 107).

En conclusion, je présenterai des pistes de réflexion inspirées des travaux de Maalouf (1998; 2009) qui pourront être pertinentes pour contrer l'insécurité linguistique. 


\section{Communautés francophones et immigration canadienne}

Au Canada, les familles viennent de partout dans le monde et les rapports au français sont aussi diversifiés que la composition démographique du pays (Richards). Plus de 10,4 millions de personnes peuvent soutenir une conversation en français (hors Québec, 2,7 millions) (Statistique Canada 2017a). À l'extérieur du Québec, en 2016, le nombre d'individus ayant déclaré le français comme langue maternelle était de 3,8 \%; une légère diminution en comparaison à 2011 (soit $4 \%$ ). De plus, la population ayant le français comme première langue officielle parlée réside essentiellement au Nouveau-Brunswick (31,8 \%), au Yukon (4,6 \%) et en Ontario (4,1\%) (Statistique Canada 2017a).

\subsection{L’Ontario français}

À la frontière du Québec, il n'existe pas une communauté franco-ontarienne identique (Remysen), car elles se sont construites au fil des décennies avec les flux migratoires francophones. Plus précisément

[...] les francophones habitent des îles différentes, avec des populations aux accents mélangés, aux identités multiples, aux caractéristiques rurales ou urbaines, aux racines d'ici ou d'ailleurs. [...] Les francophones vivent une réalité fragmentée; ils sont éparpillés un peu partout; ils habitent un archipel. Ils ont une identité locale, régionale, nationale, internationale (Cardinal 72).

En 2016, la population totale ontarienne était de 13,3 millions. Le nombre de francophones (première langue officielle parlée) était de 504130 et le nombre de personnes qui avait l'anglais et le français comme premières langues officielles parlées était de 92940 (Statistique Canada 2017f). Le bilinguisme, au sein des familles francophones, est une réalité largement majoritaire. En 2011, sept couples sur dix avec enfant(s) sont exogames (soit 68, $3 \%$ ), c'est-à-dire qu'un des parents est francophone et que l'autre ne l'est pas (Statistique Canada 2011). En outre, en 2016, 77 \% des francophones parlent plus d'une langue à la maison (français avec anglais ou avec une autre langue), comparativement à $23 \%$ d'entre eux qui parlent seulement français à la maison (Statistique Canada 2016).

\subsection{L'immigration canadienne}

Concernant le portrait multiculturel du pays, au Canada, plus de 200 langues maternelles ont été déclarées lors du recensement populationnel de 2016 (Statistique Canada 2017b). Qui plus est, 7,5 millions de personnes nées à l'étranger résident au Canada, soit plus d'une personne sur cinq. De 2011 à 2016, 1,2 million d'immigrants sont arrivés au Canada en provenance principalement des Philippines, de l'Inde et de la Chine (465 015 personnes) (Statistique 
Canada 2017b). Cependant, la diversité culturelle et linguistique se reflète essentiellement dans les métropoles canadiennes. En 2016, 75,5\% des personnes immigrantes résident à Toronto, Vancouver, Calgary, Edmonton, Montréal et Ottawa-Gatineau (Statistique Canada 2017d). Plus précisément, Toronto a le nombre d'immigrants le plus élevé au Canada avec 46,1\% de sa population, suivi de Vancouver (40,8 \%) et Calgary (29,4\%) (Statistique Canada 2017e). Bref, la diversité culturelle et linguistique au Canada est une réalité très circonscrite géographiquement.

Ajoutons que $76,4 \%$ des immigrants parlent plus d'une langue, comparativement à $27,5 \%$ des Canadiens de naissance (Statistique Canada 2017c). Ils ont déclaré à plus de $70 \%$, lors du dernier recensement de 2016, avoir une langue maternelle autre que le français ou l'anglais. Toutefois, $93,2 \%$ des immigrants connaissent l'une des deux langues officielles.

L'immigration francophone est en enjeu important pour la vitalisation des communautés francophones hors Québec. Selon Statistique Canada $(2011,9)$ : " outre un faible taux de fécondité et une transmission incomplète de la langue maternelle française des parents aux enfants, c'est l'immigration internationale qui influe le plus sur l'évolution du français au Canada ». Par exemple, de 2011 à 2016, les immigrants ayant le français comme langue maternelle s'établissent majoritairement au Québec (seulement 1,4\% ailleurs au Canada; Statistique Canada 2017c). Dans le cadre de cet article, je n'aborde pas en détail les enjeux et défis de l'immigration francophone hors Québec, mais les immigrants qui choisissent de s'établir dans une province anglophone canadienne sont rapidement plongés dans les rapports de force entre francophones et anglophones. Il existe aussi des rapports de force entre francophones que les immigrants découvriront (par exemple, les rapports de force autour de la norme française et de la qualité de la langue parlée et écrite en Ontario français).

\section{Langue, identité, mondialisation}

J'ai déjà écrit ailleurs l'évolution du concept de l'identité et sa nature polysémique (Bergeron). Je n’y reviendrai pas en détail, mais j'aimerais l'aborder sous l'angle de la triade «langue, identité, mondialisation». La question identitaire est fortement associée à la langue française au Canada (Armand, Dagenais et Nicollin). Cependant, « le rapport à l'identité d'un individu peut être complexe, surtout quand celui-ci est confronté à une réalité environnante qui ne lui permet pas de vivre constamment dans la langue et la culture de ses ancêtres » (Gérin-Lajoie 150). C'est le cas pour les individus qui n'ont pas la langue anglaise comme langue maternelle. En outre, cette triade pose un défi supplémentaire lorsque d'autres francophones issus de la mondialisation arrivent au Canada. Ce 
ne sont pas tous les immigrants francophones qui se définissent par la langue, car ces derniers ont parfois une autre langue maternelle, sans oublier ceux qui sont plurilingues et francophones. Enfin, il y a des francophones qui ont vécu dans un contexte majoritaire dans leur pays ou leur province et qui doivent maintenant s'adapter à une réalité majoritaire anglophone.

Spécifions que l'identité individuelle, «c'est ce qui fait que je ne suis identique à aucune autre personne » (Les identités meurtrières 16). L'identité est un processus individuel de construction psychosociale, mais inscrite dans un contexte sociohistorique influencé par la mondialisation, voire enracinée dans une certaine forme d'américanisation du marché linguistique, culturel et économique (Hagège). Le pouvoir symbolique d'imposer une vision du monde à travers le médium de la mondialisation s'impose, en quelque sorte, aux individus, mais «à travers des principes de di-vision» (Langage et pouvoir 283). Par exemple, cette triade est propice aux luttes identitaires, car « la langue, en l'occurrence, joue le rôle d'une révélatrice. Elle exprime, rend sensible ou cristallise un grand nombre de problèmes sociaux » (Klinkenberg 13). En somme, la triade «langue, identité, mondialisation» est au cœur du processus identitaire des francophones canadiens qui vivent dans un contexte linguistique minoritaire. Enfin, cette triade suscite un autre débat sur le français normé.

\subsection{La norme linguistique}

Une norme linguistique peut engendrer des problèmes sociaux lorsqu'elle devient hégémonique. D’une manière précise, une norme linguistique hégémonique trouve sa légitimité dans certaines formes linguistiques "(dites "langue standard, légitime, commune, soignée, correcte, classique, littéraire, soutenue, etc.”) » (Blanchet, Clerc et Rispail 2014, 290) qui seraient reconnues meilleures que d'autres formes linguistiques (langue régionaliste, métissée avec d'autres langues, etc.). Cette norme linguistique est normative dans la mesure qu'il «s'agit d'un idéal de langue, d'une fiction de langue une, homogène, monolithique, donc d'une langue idéale, idéalisée » (Houdebine 1993, 33). En d'autres mots, la norme est une "intervention normative sur l'usage (il faut parler comme ceci et non pas comme cela)» (Calvet 1999, 153). En conséquence, toute déviation par rapport à la norme peut conduire à des formes de discriminations linguistiques, car il n'y a qu'une langue "approuvée » et enracinée dans une idéologie hégémonique (Blanchet, Clerc et Rispail; Boyer). Cette idéologie peut engendrer de l'insécurité linguistique chez ceux qui ont conscience de ne pas maîtriser la norme linguistique ou lorsqu'un individu ou des groupes d'individus leur disent qu'ils ne la maitrisent pas. En contexte linguistique minoritaire, la protection de la langue française et les risques d'assimilation sont toujours d'actualité. En conséquence, l'idéologie 
hégémonique de la norme linguistique — en cherchant à défendre et à protéger la «qualité » de la langue française — engendre (malgré elle) de l'insécurité linguistique.

\section{L'insécurité linguistique}

Il est important de préciser qu'il existe des degrés d'insécurité : «il n’y a pas sécurité ou insécurité, il y a 20,30,60 ou $80 \%$ de sécurité » (Calvet 172). Cette précision faite, en contexte minoritaire francophone, les personnes qui semblent les plus affectées sont les filles et les jeunes femmes (Desabrais) et cette insécurité peut perturber autant l'apprenant du français que les francophones en milieu minoritaire. De plus, elle peut être ressentie à l'oral comme à l'écrit, mais c'est davantage dans les situations de communication orale qu'elle est plus prononcée (Lamoureux; Lozon). En conséquence, les performances scolaires des élèves sont affectées négativement (Boudreau, Dubois et d'Entremont) et l'insécurité suscite même une remise en question de l'identité et des compétences linguistiques (Hinch et Larouche). Enfin, l'insécurité linguistique agit sur les pratiques linguistiques et « influence le choix de parler telle langue plutôt que telle autre ou telle variété plutôt que telle autre, la décision de prendre la parole ou de se taire, la mise en scène de traits stigmatisés comme emblème identitaire ou, encore, l'occultation de ces mêmes traits par peur du ridicule » (Dubois, Boudreau et d'Entremont, cités dans Desabrais 125). Ultimement, l'insécurité linguistique peut conduire à l'assimilation à la langue anglaise et au décrochage scolaire ou culturel (Desabrais).

Il existe plusieurs définitions de l'insécurité linguistique dans la francophonie. D’une manière générale, cette notion correspond «à la conscience qu'il existe une norme exogène, que l'on associe à une région extérieure, qui serait supérieure par rapport à la variété linguistique en usage dans sa propre région » (Gérin-Lajoie et Labrie 87). Selon Bretegnier et Ledegen (9), cette insécurité est "l'expression d'un sentiment d'exclusion, d'extériorité, d'exogénéité, comme quête d'admission, de communauté, de légitimité linguistique et identitaire. » En lien avec la norme, l'insécurité linguistique « est maximale dans les groupes qui ont à la fois des pratiques faiblement conformes en même temps qu'une conscience aiguë de la norme » (Klinkenberg 48). Alors que dans les institutions d'enseignement, l'insécurité linguistique d'un individu

[...] dépend de sa connaissance de la langue légitime et de la conscience qu'elle a d'une distance entre la norme scolaire et ses façons de parler, deux "savoirs » produits avec beaucoup de constance par l'institution scolaire qui en fait sa priorité (la langue de scolarisation étant moyen d'enseignement-apprentissage dans toutes les disciplines, elle fait l'objet d'une évaluation constante) (Blanchet, Clerc et Rispail 294). 
Comme d'autres auteurs canadiens (LeBlanc; Remysen), je vais utiliser les trois types d'insécurisation de Calvet pour étudier l'insécurité linguistique en contexte minoritaire francophone :

1) Insécurisation formelle: le discours de l'autre, la correction sociale, la rétroaction font comprendre au locuteur qu'il "parle mal». Ce cas de figure est fréquent, facile à observer.

2) Insécurisation statutaire: le discours social, l'idéologie dominante font croire au locuteur que la langue qu'il parle a moins de valeur que d'autres formes linguistiques en présence, qu'il utilise, par exemple, un dialecte ou un patois et non pas une langue.

3) Insécurisation identitaire: le groupe, la communauté, font sentir au locuteur qu'il ne parle pas comme l'un des leurs à cause de ce qu'il parle ou de la façon dont il parle (Calvet 172).

L'insécurisation formelle (« rapport du nombre de locuteurs déclarant parler de telle manière au nombre d'entre eux pensant qu'il faut parler de telle manière », Calvet 1999, 168) ainsi que l'insécurisation identitaire du locuteur ("qui voudrait ou pense appartenir à un groupe donné », Calvet 172) seront étudiées à travers le prisme des accents francophones.

\subsection{Exemples d'insécurisation formelle et identitaire en Ontario français}

Sophie Albert est enseignante à l'École secondaire Roméo-Dallaire (Barrie, Ontario). Elle est native du nord de l'Ontario (Blind River) de deux parents francophones. Essentiellement, les valeurs familiales étaient de «parler correctement et continuellement en français ». En 2004, elle entreprend un programme d'étude en éducation à l'Université laurentienne (Sudbury). Elle réalise rapidement qu'elle est « la seule » avec un accent différent (insécurisation formelle). Plus précisément, elle affirme que : "personne ne m'avait préparée, personne ne m'avait donné de signe qu'un jour je me sentirais exclue de ma propre communauté » (insécurisation identitaire). De plus, « les commentaires comme "tu parles pas mal bien pour une fille du Sud" m’ont donné l'impression que les gens trouvaient que mon accent était laid et tout à coup, j'avais honte de mon français» (Albert 45). La honte ressentie est justement l'une des manifestations de l'insécurité linguistique. Poursuivons avec un autre exemple d'insécurisation formelle. Cortney Lacroix est originaire de Midland (Ontario). Elle se résout à parler uniquement en anglais, car elle est souvent jugée sur son accent par d'autres francophones. Elle dit ceci: «avec mes amis, je suis confortable [en français]. Mais quand je vais à Ottawa, on me regarde drôlement et on me juge. On me fait sentir mal, comme si mon accent n'était pas correct » (citée dans Fortin-Gauthier, n. p.). Selon Jérémie Spadafora, président de la Fédération de la jeunesse franco-ontarienne, «l'insécurité linguistique est un 
phénomène très commun. Et après un certain temps à se faire critiquer, certains jeunes décident de simplement parler en anglais, une langue où les différents accents semblent être moins problématiques » (cité dans Fortin-Gauthier n. p.). D'ailleurs, selon François Boileau, Commissaire aux services en français de l'Ontario, «il y a des francophones qui hésitent à demander des services en français parce que le français de leur interlocuteur va être meilleur que le leur, c'est ce qu'ils pensent» (cité dans Radio-Canada 2017, n. p.). L'insécurité linguistique se manifeste de différentes manières, dont l'insécurisation formelle et identitaire au sein des communautés francophones. Il existe aussi différentes manières de sensibiliser la population à cette problématique et l'humour en est un moyen.

\section{Vidéo d'Improtéine}

En guise d'illustration des enjeux linguistiques et identitaires chez les FrancoOntariens, le groupe humoristique Improtéine a réalisé, en 2014, une capsule vidéo. Cette capsule permet de comprendre rapidement le pluralisme identitaire en Ontario français et le rôle des accents. Je transcris quelques extraits de la vidéo $^{1}$, L'accent franco-ontarien standardise $^{2}$, afin d'accompagner la discussion à la section suivante :

En 11 ans de tournée, Improtéine s'est rendu compte de trois choses : 1) en Acadie, ils ont un accent particulier, c'est charmant, puis tout le monde les reconnaît, 2) au Québec, ils ont un accent particulier, c'est charmant, puis tout le monde les reconnaît, 3) en Ontario français, on n'a pas d'accent particulier, on est loin d'être charmant, puis personne [ne] nous reconnait.

Puis ça, c'est un problème!

Pour que les Franco-Ontariens se démarquent, ça nous prendrait un petit quelque chose d'unique. Parce qu'en Ontario français, on ne peut pas vraiment parler de notre accent; il change tellement dépendamment de la région. [...]

Bon, comment on règle ça? Facile, on standardise l'accent franco-ontarien. On se dit qu'à partir de maintenant, tous les Franco-Ontariens parlent avec le même accent. Lequel? Celui du Nord? Bien non, on veut avoir un peu de classe. Celui de l'Ouest? Bien non, on veut quand même se faire comprendre. De l'Est? On va encore se faire prendre pour des Québécois. Thunder Bay? Ha, ha, ha! Non, non, je pense que l'accent du Sud, ce serait parfait. Genre, Hamilton? Ou Burlington! C'est assez différent pour se faire remarquer. Oui, oui, c'est du français, mais avec une certaine mélodie. [...]

Puis imaginez comment on serait bien, si tous les Franco-Ontariens parlaient avec le même accent. C’est vrai ça! [...]

[Deux personnes se rencontrent] - Bonjour! C'est vraiment beau [devant une peinture]. Est-ce que tu es Franco-Ontarien? - Oui, est-ce que tu es Franco-Ontarien? - Oui. - Oh, mon Dieu! Tu arrives d'où? - De Hearst [ville] et toi? - De l'épicerie [un marché]. - As-

1 Le lecteur est invité à écouter la vidéo (3 minutes) et à poursuivre ensuite la lecture : https://www.youtube.com/watch?v=2Uj p2kNzI8

${ }^{2}$ Le Groupe média TFO m'autorise à utiliser, dans cet article, un lien internet vers cette vidéo YouTube. 
tu vu le match de sport hier? - Le sport avec le ballon? - Non, le sport avec le bâton. Ha, oui!

[Une personne arrive pour participer à la discussion, mais avec un accent différent] Bonjour les compagnons! Comment allez-vous? [Les deux autres personnes répondent] - Booooou! [L'autre personne quitte triste]

Le nouvel accent, ça va sauver les Franco-Ontariens! Il y a des gens qui penseraient que notre accent standardisé, c'est un pas vers l'assimilation. Au contraire, c'est la prise en main d'un peuple. Le ralliement d'une société. Le reflet d'une culture. Les FrancoOntariens seraient enfin fiers de parler français entre eux. On pourrait crier tous ensemble, d'une même voix : on existe! On existe!

\section{Discussion}

Cette capsule humoristique met en valeur le pluralisme identitaire au prisme des différents accents existants en Ontario français. Il met aussi en valeur l'insécurisation formelle et identitaire des communautés plurielles francoontariennes. Selon Olivier Nadon ${ }^{3}$, l'un des humoristes de la vidéo, « la notion d'adopter l'accent du sud de la province avec ses sonorités et ses chansons anglophones, comme accent standard des Franco-Ontariens représente bien cette idée des composantes multiples de la culture.»Cependant, il ajoute qu'«imposer l'accent standardisé à TOUS les Franco-Ontariens est ridicule, mais l'accent témoigne tout de même d'un équilibre entre les diverses cultures à l'œuvre dans nos communautés. »

Vouloir imposer une «idéologie hégémonique » à l'aide d'un nouvel accent standardisé serait de mettre en valeur une forme d'expression de la langue française au détriment des autres formes existantes. En d'autres mots, le désir d'être reconnu (sécurisation identitaire) ne doit pas être recherché en établissant une nouvelle norme linguistique du parler français, ce qui reviendrait à accroître le sentiment d'insécurité linguistique (insécurisation formelle et identitaire), tel qu'observé dans la vidéo (celui qui est exclu en raison qu'il n'a pas l'accent standardisé). De fait, «l'insécurité linguistique est la manifestation d'une quête non réussie de légitimité » (Francard 13).

Au sujet des accents et de l'absence de légitimité en Ontario français, et ce, en comparaison avec le Québec et le Nouveau-Brunswick (extrait de la vidéo : « en Ontario français, on n'a pas d'accent particulier, on est loin d'être charmant, puis personne [ne] nous reconnait »), le musicien franco-ontarien, Damien Robitaille, affirme :

Je vis les préjugés sur l'accent à tous les jours. Ce sont des gens ignorants qui font cela. Au départ, quand je suis arrivé au Québec, ça me rendait inconfortable. Mais avec le temps, j’ai réalisé que c'était une force. Il faut être fier de ce qu'on a d'unique. Souvent

3 Olivier Nadon m’a autorisé à utiliser cette partie des courriels échangés avec lui le 20 janvier 2016. 
les gens ont peur de quelque chose qui est différent. Mais je crois que les FrancoOntariens devraient encore plus exploiter leur accent. Je crois qu'il faut embrasser chacun des accents, car c'est ça qui rend une langue riche (cité dans Fortin-Gauthier n. p.).

Les propos de Damien Robitaille reflètent l'importance de la diversité linguistique pour contrer l'insécurité linguistique, dont l'insécurisation formelle. Comme l'indique Calvet, il existe une graduation de sécurité/insécurité linguistique chez les individus. De fait, «chaque personne va réagir différemment. Certains vont vouloir s'affirmer davantage, d'autres vont ajuster leur façon de s'exprimer et enfin, il y en a qui vont éviter de parler le français » (Freynet citée dans Vachet, n. p.).

Afin de lutter contre l'insécurité linguistique, je propose une piste de réflexion inspirée de l'académicien français et libanais, Amin Maalouf (Les identités meurtrières; Le dérèglement du monde). Tout d'abord, d'un point de vue individuel : «c'est notre regard qui enferme souvent les autres dans leurs plus étroites appartenances, et c'est notre regard aussi qui peut les libérer» (Les identités meurtrières 29). Il est important de préciser que «faire semblant d'ignorer les différences physiques ou culturelles serait absurde; mais on passerait à côté de l'essentiel si on se limitait aux différences les plus manifestes au lieu d'aller plus loin, vers la personne elle-même, dans son individualité » (Le dérèglement $d u$ monde 239). A priori, le changement social vers une plus grande inclusion de la diversité linguistique et culturelle passe par une prise de conscience individuelle et citoyenne de l'existence de l'Autre, du développement de l'altérité dans les communautés francophones.

L'accent d'une langue est en quelque sorte un médium, un marqueur important pour les individus, surtout pour ceux en quête de légitimité dans un contexte minoritaire. Le besoin de sécurité identitaire est bien ancré dans la réalité de notre contemporanéité, c'est-à-dire que la construction de l'« identité $[\ldots]$ serait perçue comme la somme de toutes nos appartenances, et au sein de laquelle l'appartenance à la communauté humaine prendrait de plus en plus d'importance » (Les identités meurtrières 115). Les liens entre l'identité individuelle et la culture issue de la mondialisation sont indéniablement interdépendants, comme nous l'avons vu précédemment. En somme, une expression de Maalouf (Le dérèglement du monde 263) résume bien cette recherche d'équilibre entre individus différents, qui partagent une même communauté humaine: "vous pourrez devenir l'un des nôtres, pleinement, sans cesser d'être vous-même. » Il est important de préserver la diversité des accents pour sécuriser les identités plurielles francophones dans un contexte minoritaire.

\section{Conclusion}


Dans le cadre de cet article, $j$ 'ai dressé un portrait général de l'Ontario français et des défis de la reconnaissance des diversités linguistiques chez les communautés francophones. Les Franco-Ontariens sont majoritairement bilingues et vivent en plus grand nombre dans le nord-est et l'est de l'Ontario (frontière avec le Québec). La transmission de la langue française ainsi que l'immigration francophone sont des enjeux importants pour la vitalisation des communautés francophones, sachant que ces dernières vivent dans un contexte minoritaire et pluriel.

L'absence de reconnaissance de cette diversité culturelle et identitaire peut conduire à des pratiques discriminatoires, dont les conséquences sont, entre autres, l'insécurité linguistique. La norme linguistique, une et indivisible, du français joue un rôle important dans cette forme d'insécurité. De plus, « la langue se révèle tantôt source de fierté et de solidarité dans sa dimension identitaire, tantôt source de honte quand elle est confrontée à d'autres locuteurs qui la partagent » (Boudreau et Dubois 147). Selon Maalouf (Les identités meurtrières), de toutes les appartenances identitaires que nous nous reconnaissons, la langue est presque toujours l'une des plus importantes. Or, l'insécurité linguistique est une donnée importante dans la compréhension des comportements et des pratiques linguistiques en contexte minoritaire (Dubois, Boudreau et d'Entremont, cités dans Desabrais).

D'une manière humoristique, le groupe Improtéine nous éclaire sur la recherche d'équilibre entre la diversité linguistique et l'appartenance à la francophonie plurielle ontarienne. La recherche d'une légitimité, d'un statut identitaire, est apparente dans la volonté d'être reconnu à l'aide d'un accent standardisé, c'est-à-dire normé. Cependant, l'insécurisation formelle n'est pas la solution; le pluralisme linguistique francophone doit s'exprimer librement et obtenir une légitimité sans emprunter le chemin de la discrimination. Pour se faire, il faut (ré) interroger la norme linguistique en vigueur en Ontario français afin qu'elle reflète la réalité contemporaine dite plurielle des communautés francophones. Cela dit, avant d'être en mesure de (ré)interroger la norme linguistique, il faudrait une prise de conscience collective sur l'idéologie hégémonique de la norme linguistique. Les discriminations basées sur les accents peuvent être repérées facilement dans les communautés (Calvet; Lippi-Green). Ce type de discrimination, basé sur l'idéologie hégémonique de la norme linguistique, est généralement accepté et perçu comme approprié (hypercorrection de la langue, chasser les menaces à la langue, etc.) dans la protection d'une langue (Lippi-Green). Toutefois, les individus «ne réalisent pas que chercher la "pureté de la langue", c'est chercher une "pureté", c'est refuser les contacts, les mélanges, les métissages, c'est refuser la vie sociale» (Blanchet 164). 
En conclusion, je n'ai pas présenté la totalité des dynamiques identitaires de l'Ontario français, à savoir l'influence de la langue, de l'histoire, de l'ethnicité ainsi que de la culture sur le dynamisme des communautés francophones. J'ai donc proposé quelques pistes de réflexion - sans viser la stigmatisation de l'Ontario français ou des communautés anglophones et francophones canadiennes - à partir de certains comportements épilinguistiques, et ce, dans l'objectif de contribuer à la réflexion sur l'insécurité linguistique au Canada. À ce jour, il est très difficile d'avoir une connaissance étendue de cette problématique dans la population. Il serait alors important de développer des outils afin d'étudier cette forme d'insécurité ainsi que d'autres discriminations (glottophobie; cf. Blanchet) que vivent les populations francophones canadiennes. 


\section{Bibliographie}

Albert, Sophie. Du Nord au Sud, d'élève à enseignante : trajectoires d'une francophone du Centre-Sud, Prendre sa place. Parcours et trajectoires identitaires en Ontario français, édité par Sylvie A. Lamoureux et Megan Cot., Les Éditions David, 2012, pp. 45-50.

Armand, Françoise, Diane Dagenais et Laura Nicollin. «La dimension linguistique des enjeux interculturels : de l'Éveil aux langues à l'éducation plurilingue. » Éducation et francophonie, vol. 36, n 1, 2008, pp. 44-64.

Bergeron, Christian. «L'autonomie dans la modernité avancée : ouverture des possibles... et nouvelles responsabilités. »L'Observatoire, vol. 88, 2016, pp. 5-7.

Blanchet, Philippe. Discriminations : combattre la glottophobie, Éditions Textuel, 2016.

Blanchet, Philippe, Stéphanie Clerc et Marielle Rispail. «Réduire l'insécurité linguistique des élèves par une transposition didactique de la pluralité sociolinguistique. Pour de nouvelles perspectives sociodidactiques avec l'exemple du Maghreb. » Ela. Études de linguistique appliquée, vol. 3, n 175, 2014, pp. 283-302.

Boudreau, Annette, Dubois, Lise et d'Entremont, Véronica. «Représentations, sécurité/insécurité linguistique et éducation en milieu minoritaire. » Francophonie, minorités et pédagogie, édité par Sylvie Roy et Phyllis Dalley, Les Presses de l'Université d'Ottawa, 2008, pp. 145-176.

Boudreau, Annette et Dubois, Lise. «J'parle pas comme les Français de France, ben c'est du français pareil; j'ai ma own p'tite langue ». L'insécurité linguistique dans les communautés francophones périphériques, édité par Michel Francard, Geneviève Geron et Régine Wilmet, Actes du colloque de Louvain-La-Neuve, 10-12 novembre 1993, Vol. I : Cahiers de l'Institut de Linguistique de Louvain, vol. 19, n ${ }^{\circ} 3-4,1993$, pp. 147-168.

Bourdieu, Pierre. Langage et pouvoir symbolique, Éditions du Seuil, 2001.

---. Ce que parler veut dire, Fayard, 1982.

Boyer, Henri. Introduction à la sociolinguistique, Éditions Dunod, 2017.

Bretegnier, Aude et Gudrun Ledegen. Sécurité, insécurité linguistique. Terrains et approches diversifiées, propositions théoriques et méthodologiques, Éditions L'Harmattan. 2002.

Calvet, Louis-Jean. Pour une écologie des langues du monde, Éditions Plon, 1999.

Cardinal, Linda. "Ruptures et fragmentations de l'identité francophone en milieu minoritaire; un bilan critique. » Sociologie et sociétés, vol. 26, n 1, 1994, pp. 71-86.

Dalley, Phyllis. «Diversité linguistique et attitude polynomiste en formation à l'enseignement ». Des paroles, des langues et des pouvoirs, édité par Romain Colonna, Éditions L'Harmattan, 2014, pp. 151-168. 
Desabrais, Tina. Les mots pour le dire... L'influence de l'(in) sécurité linguistique sur l'expérience d'étudiantes de milieux francophones minoritaires canadiens inscrites aux études supérieures à l'Université d'Ottawa, Thèse en éducation, Ottawa, Université d'Ottawa, 2013.

Fortin-Gauthier, Étienne. « Des jeunes Franco-Ontariens jugés en raison de leur accent». Télévision Franco-Ontarienne (TFO), 17 août 2015, https://onfr.tfo.org/des-jeunes-franco-ontariens-juges-en-raison-deleur-accent/. Consulté le 4 février 2017.

Francard, Michel. L'insécurité linguistique en Communauté française de Belgique. Bruxelles, Ministère de la Culture, Service de la Langue française, 1993.

Gérin-Lajoie, Diane. « Mieux comprendre le rapport à l'identité ». Prendre sa place. Parcours et trajectoires identitaires en Ontario français, édité par Sylvie A. Lamoureux et Megan Cotnam, Les Éditions David, 2012, pp. 149-154.

Gérin-Lajoie, Diane et Normand Labrie. «Les résultats aux tests de lecture et d'écriture en 1993-1994 : une interprétation sociolinguistique », L'enjeu de la langue en Ontario francais, édité par Normand Labrie et Gilles Forlot, Éditions Prise de parole, 1999, pp. 79-109.

Hagège, Claude. Contre la pensée unique, Éditions Odile Jacob, 2012.

Hinch, Chloé et Fréderic Larouche, «Bâtir sur le roc : prévenir et lutter contre l'insécurité linguistique. » $65^{\mathrm{e}}$ congrès de l'Association canadienne d'éducation de langue française (ACELF), septembre 2012. http://www.acelf.ca/c/fichiers/Actes12 A4 C10.pdf Consulté le 4 février 2017.

Houdebine, Anne-Marie. «De l'imaginaire des locuteurs et de la dynamique linguistique. Aspects théoriques et méthodologiques.» L'insécurité linguistique dans les communautés francophones périphériques, édité par Michel Francard, Geneviève Geron et Régine Wilmet. Actes du colloque de Louvain-La-Neuve, 10-12 novembre 1993, Vol. I : Cahiers de l'Institut de Linguistique de Louvain, vol. 19, n 3-4, 1993, pp. 31-40.

Klinkenberg, Jean-Marie. La langue dans la cité. Vivre et penser l'équité culturelle, Les Impressions Nouvelles, 2015.

Labrie, Elisabeth. «La construction identitaire dans la francophonie ontarienne : le cas des franco-majoritaires. » Prendre sa place. Parcours et trajectoires identitaires en Ontario français, édité par Sylvie A. Lamoureux et Megan Cotnam, Les Éditions David, 2012, pp. 23-44.

Lamoureux, Sylvie A. "L'expérience étudiante au Régime d'immersion en français : perspectives et constats. » Cabiers de l'ILOB, nº 6, 2013, pp. 109121.

Leblanc, Matthieu. «Le français, langue minoritaire, en milieu de travail : des représentations linguistiques à l'insécurité linguistique.» Nouvelles perspectives en sciences sociales, vol. 6, $\mathrm{n}^{\circ}$ 1, 2010, pp. 17-63. 
Lippi-Green, Rosina. English with an Accent. Language, Ideology, and Discrimination in the United States. 2nd ed., Routledge, 2012.

Lozon, Roger. "Les jeunes du Sud-Ouest ontarien: représentation et sentiments linguistiques. » Francophonies d'Amérique, no 12, 2001, pp. 83-92.

Maalouf, Amin. Le dérèglement du monde. Quand nos civilisations s'épuisent, Éditions Grasset \& Fasquelle, 2009.

---. Les identités meurtrières, Éditions Grasset \& Fasquelle, 1998.

Radio-Canada. "Lutter contre l'insécurité linguistique des francophones». Radio-Canada Windsor, 10 mai 2017, http://ici.radiocanada.ca/nouvelle/1033013/insecurite-linguistique-chez-francophones. Consulté le 20 septembre 2017.

Richards, Mary. «Les jeunes, la mondialisation, le bilinguisme et le milieu scolaire francophone. » Prendre sa place. Parcours et trajectoires identitaires en Ontario français, édité par Sylvie A. Lamoureux et Megan Cotnam, Les Éditions David, 2012, pp. 85-106.

Remysen, Wim. «L'insécurité linguistique des Francophones Ontariens et NéoBrunswickois. Contribution à l'étude de la francophonie canadienne. » Aspects de la nouvelle francophonie canadienne, édité par Simon Langlois et Jocelyn Letourneau, Presses de l’Université Laval, 2004, pp. 95-116.

Statistique Canada. Le français, l'anglais et les minorités de langue officielle au Canada. Recensement de la population, 2016. 31 août 2017a, http://www12.statcan.gc.ca/census-recensement/2016/as-sa/98-200x/2016011/98-200-x2016011-fra.cfm. Consulté le 25 mars 2018.

---. Population des immigrants au Canada. 25 octobre 2017b, https://www.statcan.gc.ca/pub/11-627-m/11-627-m2017028-fra.htm. Consulté le 25 mars 2018.

---. L'intégration linguistique des immigrants et les populations de langue officielle au Canada. Recensement de la population, 2016. 25 octobre 2017c, http://www12.statcan.gc.ca/census-recensement/2016/as-sa/98-200x/2016017/98-200-x2016017-fra.pdf. Consulté le 25 mars 2018.

---. Diversité linguistique et plurilinguisme au sein des foyers canadiens. Recensement de la population, 2016. 31 août 2017d, http://www12.statcan.gc.ca/censusrecensement/2016/as-sa/98-200-x/2016010/98-200-x2016010-fra.pdf.

Consulté le 26 mars 2018.

---. Immigration et diversité ethnoculturelle - Faits saillants en tableaux. 1 novembre 2017e, http://www12.statcan.gc.ca/census-recensement/2016/dp$\mathrm{pd} / \mathrm{hlt}-$

fst $/$ imm/Tableau.cfm?Lang $=F \& T=12 \& \mathrm{Geo}=00 \& v i e w=2 \& a g e=1 \&$ sex $\equiv 1 \& \mathrm{SO}=16 \mathrm{D} \& \mathrm{SP}=1$. Consulté le 26 mars 2018.

---. Profil du recensement, Recensement de 2016. 29 novembre 2017f, http://www12.statcan.gc.ca/census-recensement/2016/dp$\mathrm{pd} / \mathrm{prof} /$ details $/$ page.cfm?Lang $=\mathrm{F} \& \mathrm{Geo} 1=\mathrm{PR} \&$ Code $1=35 \& \mathrm{Geo} 2=\mathrm{PR}$ 
$\underline{\& \text { Code } 2=01 \& \text { Data }=\text { Count } \& \text { Search Text }=\text { Ontario } \& \text { Search Type }=\text { Begins }}$ $\&$ SearchPR $=01 \& B 1=$ Language $\&$ TABID $=1$. Consulté le 26 mars 2018.

---. Census 2016 Topic-Based Tabulations. Aggregate Data. Tables for Language (98-400X2016345). Beyond 2020 (base de données), 2016. http://odesidownload.scholarsportal.info/documentation/CENSUS/20 16/cen16language.html. Consulté le 26 mars 2018.

---. Le français et la francophonie au Canada. 2011, http://www12.statcan.gc.ca/census-recensement/2011/as-sa/98-314x/98-314-x2011003 1-fra.pdf. Consulté le 10 mars 2017.

Vachet, Benjamin. "La discrimination des accents, une menace pour le français. » Télévision Franco-Ontarienne (TFO). 2 mars 2018, https://onfr.tfo.org/la-discrimination-des-accents-une-menace-pour-lefrancais/. Consulté le 10 mars 2018.

Vaillancourt, Richard. Plan en construction identitaire francophone. Pour le Conseil scolaire Centre-Nord et ses écoles élémentaires et secondaires. Edmonton, Conseil scolaire Centre-Nord, 2008 , https://centrenord.ab.ca/fichiers/cscn/Conseil/Rapports/Vision2020/CIAvril2008.pdf. Consulté le 10 septembre 2017. 